PROCEEDINGS OF THE

AMERICAN MATHEMATICAL SOCIETY

Volume 126, Number 9, September 1998, Pages 2513-2523

S 0002-9939(98)04440-2

\title{
A BOUND FOR THE DERIVED AND FRATTINI SUBGROUPS OF A PRIME-POWER GROUP
}

\author{
GRAHAM ELLIS
}

(Communicated by Ronald M. Solomon)

\begin{abstract}
This paper is based on the seemingly new observation that the Schur multiplier $M(G)$ of a $d$-generator group of prime-power order $p^{n}$ has order $|M(G)| \leq p^{d(2 n-d-1) / 2}$. We prove several related results, including sufficient conditions for a sharper bound on $|M(G)|$ to be an equality.
\end{abstract}

Let $G$ be a group with centre $Z$. Schur [13] observed that the commutator subgroup $[G, G]$ is finite whenever the quotient $G / Z$ is finite. Wiegold [17] obtained an estimate for the order of $[G, G]$ in terms of the order of $G / Z$; in particular he showed that if $G / Z$ has prime-power order $p^{n}$, then the order of $[G, G]$ is at most $p^{n(n-1) / 2}$. This bound can be attained for all $n \geq 1$, but only if $G / Z$ is elementary abelian. As a corollary, Wiegold re-derived Green's bound [7] on the order of the Schur multiplier of a prime-power group. Gaschütz, Neubüser and Yen [6] subsequently refined Wiegold's methods to obtain a sharper bound on $[G, G]$ (involving the number of generators of the quotient of $G$ by its second centre), and a corresponding sharpening of Green's bound. By a different fine tuning of Wiegold's methods, Jones ([9], [10], [11]) obtained complementary improvements in Green's bound.

In this article we reduce the above bounds on $[G, G]$ by incorporating, in turn: (1) the number of generators of $G / Z ;(2)$ the abelian group structure of the quotients of the lower central series of $G / Z$; (3) the restricted Lie algebra structure of the quotients of the lower $p$-central series of $G / Z$; (4) the "breadth in $G$ " of the preimages of the generators of $G / Z$. The fourth approach (which is the only one to involve more than the structure of $G / Z$ ) complements a result of Vaughan-Lee [16]. We obtain corresponding reductions in the above-mentioned bounds on the Schur multiplier. As an application we obtain a rough upper bound on the number of $d$-generator groups of order $p^{n}$. Furthermore, our results are presented in such a way as to yield bounds on the Frattini subgroup of a prime-power group.

Let $p$ be any prime, and let $q \geq 0$ be any nonnegative integer multiple of $p$. (We are interested mainly in the cases $q=0$ and $q=p$.) Given a group $G$ we let $Z^{q}(G)$ denote the subgroup of the centre of $G$ consisting of those elements with order dividing $q$. Given a normal subgroup $N$ in $G$, we let $N \#^{q} G$ denote the subgroup of $G$ generated by the commutators $n g n^{-1} g^{-1}$ and powers $n^{q}$ for $n \in N, g \in G$.

Received by the editors January 27, 1997.

1991 Mathematics Subject Classification. Primary 20J05.

(C)1998 American Mathematical Society 
We define

$$
\begin{array}{ll}
\gamma_{1}^{q} G=G, & \\
\gamma_{i+1}^{q} G=\left(\gamma_{i}^{q} G\right) \#{ }^{q} G, & i \geq 1, \\
\Gamma_{i}^{q} G=\gamma_{i}^{q} G / \gamma_{i+1}^{q} G, & i \geq 1 .
\end{array}
$$

Furthermore, we set

$$
\begin{aligned}
& M^{q}(G)=H_{2}\left(G, \mathbb{Z}_{q}\right), \\
& H_{n}^{q}(G)=H_{n}\left(G, \mathbb{Z}_{q}\right), \\
& R(G, q)= \begin{cases}0 & \text { if } q=0, \\
\left|\Gamma_{1}^{0} G\right| /\left|\gamma_{2}^{q}\left(M^{0}(G)\right)\right| & \text { if } q \geq p,\end{cases}
\end{aligned}
$$

where $H_{n}\left(G, \mathbb{Z}_{q}\right)$ is the $n$-th homology group of $G$ with coefficients in the abelian group $\mathbb{Z}_{q}=\mathbb{Z} / q \mathbb{Z}$. Thus $Z^{0} G$ is the centre of $G, \gamma_{1}^{0} G \leq \gamma_{2}^{0} G \leq \cdots$ is the lower central series of $G$, and when $G$ is finite $M^{0}(G)$ is the Schur multiplier of $G$. If $G$ is a finite $p$-group, then $\gamma_{2}^{p} G$ is its Frattini subgroup, and $\Gamma_{i}^{p} G$ is an elementary abelian group for $i \geq 1$. We say that a group is a " $d$-generator group" if it can be generated by $d$ elements, and by no fewer than $d$ elements.

Theorem 1. Let $G$ be any group such that $Q=G / Z^{q}(G)$ is a d-generator group of order $p^{n}$. Then:

(i) $\left|\gamma_{2}^{q} G\right| \leq p^{(d(2 n-d-1) / 2)+R(Q, q)}$.

(ii) There exists a group $\widehat{G}$ with $\widehat{G} / Z^{q}(\widehat{G}) \cong Q$ and $\left|\gamma_{2}^{q} \widehat{G}\right|=p^{(d(2 n-d-1) / 2)+R(Q, q)}$ if and only if the canonical homomorphism $H_{3}^{0}(Q) \rightarrow H_{3}^{0}\left(Q / \gamma_{i}^{p} Q\right)$ is surjective for each $i \geq 1$.

(iii) If $d=n \geq 2$, or if $d=n-1=2$, then there exists a group $\widehat{G}$ with $\widehat{G} / Z^{q}(\widehat{G})$ a d-generator group of order $p^{n}$, and such that $\left|\gamma_{2}^{q} \widehat{G}\right|=p^{(d(2 n-d-1) / 2)+R(Q, q)}$.

Note that $p^{d(2 n-d-1) / 2} \leq p^{n(n-1) / 2}$ for all integers $1 \leq d \leq n$. Thus Wiegold's bound [17, Theorem 2.1] on the commutator subgroup $\gamma_{2}^{0} G$ follows from Theorem 1.

Note that part (iii) of Theorem 1 does not extend to $d=n-2=2$. Consider, for instance, the values $p=2, q=0, n=4, d=2$ and consider any group $G$ with $G / Z^{0}(G)$ a 2-generator group of order $p^{4}$. Arguments involving the Schur multiplier (see [2]) establish the existence of such a $G$, and show that the only possibilities for the commutator subgroup are $\gamma_{2}^{0} G \cong C_{2} \times C_{2}, \gamma_{2}^{0} G \cong C_{2} \times C_{4}$ and $\gamma_{2}^{0} G \cong C_{2} \times C_{2} \times C_{4}$; in all cases $\left|\gamma_{2}^{0} G\right|$ is strictly less than $p^{d(2 n-d-1) / 2}=32$.

The next result is a variant on Theorem 1 which incorporates the structure of the abelian groups $\left(Q / Z^{0}(Q)\right)^{\text {ab }}$ and $\Gamma_{i}^{0}(Q)$ for $i \geq 1$.

Theorem 2. Let $G$ be any group with $\left|G / Z^{q}(G)\right|=p^{n}$. Set $Q=G / Z^{q}(G)$ and $\bar{Q}=Q / Z^{0}(Q)$, and suppose that

$$
\begin{aligned}
& \Gamma_{1}^{0} \bar{Q} \cong C_{p^{m_{11}^{\prime}}} \times C_{p^{m_{12}^{\prime}}} \times \cdots \times C_{p_{m_{1 r}^{\prime}}}, \\
& \Gamma_{i}^{0} Q \cong C_{p^{m_{i 1}}} \times C_{p^{m_{i 2}}} \times \cdots \times C_{p^{m_{i r}}}
\end{aligned}
$$

for $i \geq 1$. (Here $r$ is independent of $i$ and $m_{i j} \geq 0$.) Set

$$
M=\sum_{1 \leq j<k \leq r} \operatorname{minimum}\left(m_{1 j}, m_{1 k}\right)+\sum_{\substack{2 \leq i \leq r \\ 1 \leq j, k \leq r}} \operatorname{minimum}\left(m_{i j}, m_{1 k}^{\prime}\right) .
$$


Then

(i) $\left|\gamma_{2}^{q} G\right| \leq p^{M+R(Q, q)}$.

(ii) There exists a group $\widehat{G}$ with $\widehat{G} / Z^{q}(\widehat{G}) \cong Q$ and $\left|\gamma_{2}^{q} \widehat{G}\right|=p^{M+R(Q, q)}$ if the canonical homomorphism $H_{3}^{0}(Q) \rightarrow H_{3}^{0}\left(Q / \gamma_{i}^{0} Q\right)$ is surjective for each $i \geq 1$.

This theorem improves slightly on a bound of Gaschütz, Neubüser and Yen [6]. They proved that if $Q=G / Z^{0}(G)$ is a prime-power group whose central quotient $Q / Z^{0}(Q)$ is generated by $d$ elements, then

$$
|[G, G]| \leq\left|M^{0}\left(Q^{\mathrm{ab}}\right)\right||[Q, Q]|^{d} .
$$

To see the improvement, note that $M^{0}\left(Q^{\mathrm{ab}}\right)$ is just the exterior square $Q^{\mathrm{ab}} \wedge Q^{\mathrm{ab}}$ and hence that

$$
\log _{p}\left|M^{0}\left(Q^{\mathrm{ab}}\right)\right|=\sum_{1 \leq j<k \leq r} \operatorname{minimum}\left(m_{1 j}, m_{1 k}\right)
$$

Furthermore, if $Q$ is nilpotent of class $c$ say, then equality (4) below (which involves the tensor product of abelian groups) yields

$$
\begin{aligned}
\log _{p}|[Q, Q]|^{d} & =\log _{p}\left|\Gamma_{2}^{0} Q \oplus \cdots \oplus \Gamma_{c}^{0} Q\right|^{d} \\
& \geq \log _{p}\left|\left(\Gamma_{2}^{0} Q \oplus \cdots \oplus \Gamma_{c}^{0} Q\right) \otimes \Gamma_{1}^{0} \bar{Q}\right| \\
& =\sum_{\substack{2 \leq i \leq r \\
1 \leq j, k \leq r}} \operatorname{minimum}\left(m_{i j}, m_{1 k}^{\prime}\right) .
\end{aligned}
$$

The last inequality is strict precisely when the exponent of $\Gamma_{2}^{0} Q \oplus \cdots \oplus \Gamma_{c}^{0} Q$ is greater than the order of at least one of the $d$ generators of $\Gamma_{1}^{0} \bar{Q}=Q /[Q, Q] Z^{0}(Q)$; in this case the bound in Theorem 2 is lower than the bound of [6].

To illustrate the above results set $p=2, q=0$, and consider the semi-direct product $Q=\left\langle x, y \mid x^{8}=y^{4}=1, x y=y x^{3}\right\rangle$ of $C_{4}$ with $C_{8}$, which has order $2^{5}$. Let $G$ be any group with $Q \cong G / Z^{0}(G)$. Arguments involving the Schur multiplier of $Q$ (cf. [2]) show that, in this case, such a group $G$ exists and that $\gamma_{2}^{0} G$ must have order $2^{3}$. Wiegold's bound asserts that

$$
\left|\gamma_{2}^{0} G\right| \leq 2^{n(n-1) / 2}=2^{10}
$$

The bound of Gaschütz, Neubüser and Yen asserts that

$$
\left|\gamma_{2}^{0} G\right| \leq\left|M^{0}\left(C_{2} \times C_{4}\right)\right|\left|C_{2} \times C_{2}\right|^{2}=2^{5} .
$$

Since $Q$ is a 2 -generator group, Theorem $1(\mathrm{i})$ asserts that

$$
\left|\gamma_{2}^{0} G\right| \leq 2^{d(2 n-d-1) / 2}=2^{7} .
$$

Since $\gamma_{2}^{0} Q / \gamma_{3}^{0} Q \cong C_{2}, \gamma_{3}^{0} Q \cong C_{2}$, and $\gamma_{i}^{0} Q=1$ for $i \geq 4$, and since $\left(Q / Z^{0}(Q)\right)^{\text {ab }} \cong$ $C_{4} \times C_{2}$, Theorem 2(i) asserts that

$$
\left|\gamma_{2}^{0} G\right| \leq 2^{M}=2^{5} .
$$

Let $Q$ be any finite $p$-group. Then $\gamma_{c+1}^{p} Q=1$ for some $c \geq 1$, and the direct sum $\mathfrak{L}=\Gamma_{1}^{p} Q \oplus \Gamma_{2}^{p} Q \oplus \cdots \oplus \Gamma_{c}^{p} Q$ has the structure of a restricted Lie algebra over the field $\mathbb{F}=\mathbb{Z}_{p}$ (cf. [12]). The Lie bracket $\mathfrak{h}: \mathfrak{L} \times \mathfrak{L} \rightarrow \mathfrak{L}$ is induced by commutation in $Q$, and the power map $\mathfrak{p}: \mathfrak{L} \rightarrow \mathfrak{L}$ is induced by taking $p$-th powers in $Q$. For each $i \geq 1$ the bracket $\mathfrak{h}$ restricts to a linear homomorphism $\mathfrak{h}: \Gamma_{i}^{p} Q \otimes \Gamma_{1}^{p} Q \rightarrow \Gamma_{i+1}^{p} Q$ of vector spaces, and repetition of the power map $\mathfrak{p}$ yields a linear homomorphism 
$\mathfrak{p}^{i}: \Gamma_{1}^{p} Q \rightarrow \Gamma_{i+1}^{p} Q$. The bound of Theorem 1 can be improved by considering the following linear homomorphisms:

$$
\begin{aligned}
& \mathfrak{j}_{2}: \Gamma_{1}^{p} Q \otimes \Gamma_{1}^{p} Q \otimes \Gamma_{1}^{p} Q \rightarrow \Gamma_{2}^{p} Q \otimes \Gamma_{1}^{p} Q \\
& \quad x \otimes y \otimes z \mapsto \mathfrak{h}(x, y) \otimes z+\mathfrak{h}(y, z) \otimes x+\mathfrak{h}(z, x) \otimes y, \\
& \mathfrak{j}_{i}: \Gamma_{1}^{p} Q \otimes \Gamma_{1}^{p} Q \otimes \cdots \otimes \Gamma_{1}^{p} Q \rightarrow \Gamma_{i}^{p} Q \otimes \Gamma_{1}^{p} Q \\
& \quad x_{1} \otimes x_{2} \otimes \cdots \otimes x_{i+1} \mapsto\left[x_{1}, \cdots, x_{i}\right]_{l} \otimes x_{i+1}+\left[x_{i+1},\left[x_{1}, \cdots, x_{i-1}\right]_{l}\right] \\
& \quad \otimes x_{i}+\left[\left[x_{i}, x_{i+1}\right]_{r},\left[x_{1}, \cdots, x_{i-2}\right]_{l}\right] \\
& \quad \otimes x_{i-1}+\left[\left[x_{i-1}, x_{i}, x_{i+1}\right]_{r},\left[x_{1}, \cdots, x_{i-3}\right]_{l}\right] \\
& \quad \otimes x_{i-2}+\cdots+\left[x_{2}, \cdots, x_{i+1}\right]_{r} \otimes x_{1}, \\
& \mathfrak{s}_{i}: \Gamma_{1}^{p} Q \rightarrow \Gamma_{i}^{p} Q \otimes \Gamma_{1}^{p} Q, \quad x \mapsto\left(\mathfrak{p}^{i-1} x\right) \otimes x, \quad i \geq 2, \\
& \mathfrak{t}_{i}: \Gamma_{1}^{p} Q \otimes \Gamma_{1}^{p} Q \rightarrow \Gamma_{i}^{p} Q \otimes \Gamma_{1}^{p} Q, \quad x \otimes y \mapsto\left(\mathfrak{p}^{i-1} x\right) \otimes y+\left(\mathfrak{p}^{i-1} y\right) \otimes x, \quad i \geq 2
\end{aligned}
$$

Here $x_{i} \in \Gamma_{1}^{p} Q$ and we have set

$$
\begin{aligned}
{\left[x_{1}, x_{2}\right] } & =\mathfrak{h}\left(x_{1} \otimes x_{2}\right),\left[x_{1}, \cdots, x_{i}\right]_{l} \\
& =\mathfrak{h}\left(\cdots \mathfrak{h}\left(\mathfrak{h}\left(x_{1} \otimes x_{2}\right) \otimes x_{3}\right) \cdots \otimes x_{i}\right),\left[x_{1}, \cdots, x_{i}\right]_{r} \\
& =\mathfrak{h}\left(x_{1} \otimes \cdots \mathfrak{h}\left(x_{i-2} \otimes \mathfrak{h}\left(x_{i-1} \otimes x_{i}\right)\right) \cdots\right) .
\end{aligned}
$$

Note that $\mathfrak{j}_{2}(x, y, z)=0$ whenever two of $x, y, z$ are equal. Hence $\mathfrak{j}_{2}$ has trivial image whenever $Q$ is a 2-generator group.

Theorem 3. Let $G$ be any group such that $Q=G / Z^{q}(G)$ is a d-generator group of order $p^{n}$. Set

$$
a_{i}=\operatorname{dim}_{\mathbb{F}}\left(\operatorname{image}\left(\mathfrak{j}_{i}\right)+\operatorname{image}\left(\mathfrak{s}_{i}\right)+\operatorname{image}\left(\mathfrak{t}_{i}\right)\right)
$$

for $i \geq 2$, and set $a=a_{1}+a_{2}+\cdots+a_{c}$ where $\gamma_{c+1}^{p} Q=1$. Then

$$
\left|\gamma_{2}^{q} G\right| \leq p^{(d(2 n-d-1) / 2)-a+R(Q, q)} .
$$

To illustrate Theorem 3 let us return to a group $G$ such that $G / Z^{0}(G) \cong Q=$ $\left\langle x, y \mid x^{8}=y^{4}=1, x y=y x^{3}\right\rangle$. Now $\Gamma_{1}^{2} Q \cong \mathbb{Z}_{2} \oplus \mathbb{Z}_{2}$ is generated by the cosets of $x$ and $y ; \Gamma_{2}^{2} Q \cong \mathbb{Z}_{2} \oplus \mathbb{Z}_{2}$ is generated by the cosets of $x^{2}$ and $y^{2} ; \Gamma_{3}^{2} Q \cong Z_{2}$ is generated by the coset of $x^{4} ; \Gamma_{i}^{2} Q=0$ for $i \geq 4$. Since $Q$ is a 2 -generator group, $\mathfrak{j}_{2}$ has trivial image. One readily verifies that $\mathfrak{j}_{3}$ also has trivial image. Thus $a_{2}=\operatorname{dim}\left(\operatorname{image}\left(\mathfrak{s}_{2}\right)+\operatorname{image}\left(\mathfrak{t}_{2}\right)\right)=3, a_{3}=\operatorname{dim}\left(\operatorname{image}\left(\mathfrak{s}_{3}\right)+\operatorname{image}\left(\mathfrak{t}_{3}\right)\right)=1$, and $a=3+1=4$. Therefore Theorem 3 asserts that

$$
\left|\gamma_{2}^{0} G\right| \leq 2^{(d(2 n-d-1) / 2)-a}=2^{3} .
$$

Vaughan-Lee [16] showed that the commutator subgroup of a finite $p$-group of "breadth" $b$ has order at most $p^{b(b+1) / 2}$. Recall that the breadth $b(x)$ of an element $x$ in an arbitrary group $G$ is defined (with respect to the prime $p$ ) as the logarithm (to the base $p$ ) of the number of elements conjugate to $x$ :

$$
b(x)=\log _{p}|[x]| .
$$

Of course, $b(x)$ is infinite when the conjugacy class $[x]$ is infinite. The breadth of the group $G$ is defined as the maximum value of $b(x)$ for $x \in G$ if such a maximum exists; otherwise it is defined to be infinite.

Suppose that $Q=G / Z^{0}(G)$ is a finite $p$-group. If $C(x)$ denotes the centralizer of $x$ in $G$, then $p^{b(x)}=|G: C(x)|$. Since $|Q|=\left|G: Z^{0}(G)\right|=|G: C(x)| \times \mid C(x)$ : 
$Z^{0}(G) \mid$ is a power of $p$, it follows that $b(x)$ is a nonnegative integer for all $x$ in $G$. For any finite subset $X=\left\{x_{1}, \ldots, x_{d}\right\}$ in $G$, set

$$
b(X)=\left(b\left(x_{1}\right)+\cdots+b\left(x_{d}\right)\right) / d,
$$

and let $\bar{X}$ denote the image of $X$ in $Q$. It seems reasonable to define the average breadth of $G$ to be

$$
\bar{b}(G)=\operatorname{minimum}\left\{b(X): \bar{X} \text { generates } G / Z^{0}(G)\right\} .
$$

The following theorem complements Vaughan-Lee's result.

Theorem 4. Let $G$ be any group such that $Q=G / Z^{0}(G)$ is a d-generator finite p-group. If $G$ has average breadth $\bar{b}=\bar{b}(G)$, then

$$
\left|\gamma_{2}^{0} G\right| \leq p^{d \bar{b}}
$$

Our proofs of the above theorems yield the following result of independent interest.

Proposition 5. Let $Q$ be any d-generator group of order $p^{n}$.

(i) For a defined as in Theorem 3, we have

$$
\left|M^{q}(Q)\right|\left|\gamma_{2}^{q} Q\right| \leq p^{(d(2 n-d-1) / 2)-a+R(Q, q)} .
$$

(ii) If the abelian groups $\left(Q / Z^{0}(Q)\right)^{\mathrm{ab}}$ and $\Gamma_{i}^{0} Q$ have structure as in Theorem 2, then

$$
\left|M^{q}(Q)\right|\left|\gamma_{2}^{q} Q\right| \leq p^{M+R(Q, q)} .
$$

This bound is attained if the canonical homomorphism $H_{3}^{0}(Q) \rightarrow H_{3}^{0}\left(Q / \gamma_{i}^{0} Q\right)$ is surjective for each $i \geq 1$.

Proposition 5(i) (with $q=0$ ) sharpens a bound on the Schur multiplier given in [9, Corollary 2.3]. The bound in Proposition 5(ii) (with $q=0$ ) is similar to, but lower than, a bound given in [10, Theorem 4.1] (see also [11]); the latter bound uses $\gamma_{i}^{0} G /\left[\gamma_{i}^{0} G, \gamma_{i}^{0} G\right]$ in place of our $\gamma_{i}^{0} G / \gamma_{i+1}^{0} G$. Proposition 5(ii) also sharpens a bound given in $[6]$.

The proof of Proposition 5 yields analogous bounds on the exponent of $M^{q}(Q)$ and on the number of generators of $M^{q}(Q)$. Details of these are left to the reader.

Proposition 5 is relevant to the enumeration of finite $p$-groups. Let $f(n, p)$ denote the number of groups of order $p^{n}$, and set $A(n, p)=\log _{p}(f(n, p))$. Higman [8] proved that

$$
\left(2 / 27-\varepsilon_{n}\right) n^{3} \leq A(n, p) \leq\left(n^{3}-n\right) / 6
$$

where $\varepsilon_{n} \rightarrow 0$ as $n \rightarrow \infty$. Sims [14] subsequently showed that $A(n, p) / n^{3} \rightarrow$ $2 / 27$ as $n \rightarrow \infty$. In order to get a feel for the types of $p$-groups that occur one should investigate, for various group-theoretic properties $\mathcal{P}$, the number $f(n, p, \mathcal{P})$ of groups of order $p^{n}$ satisfying property $\mathcal{P}$.

Let us consider the number $f(n, p, d)$ of $d$-generator groups of order $p^{n}$, and set

$$
A(n, p, d)=\log _{p}(f(n, p, d)) .
$$


A lower bound for $A(n, p, d)$ is implicit in [8]. An upper bound can be obtained from Proposition 5. To this effect let us define

$$
\begin{aligned}
& U(n, d)= \begin{cases}(d-1)\left(n^{2}-d^{2}\right) / 2, & \text { for } d \neq 2, \\
n(n+1)-6, & \text { for } d=2,\end{cases} \\
& L(n, d)=\left(d(d+1)(n-d)-2(n-d)^{2}-2 d^{2}\right) / 2 .
\end{aligned}
$$

The following corollary shows that these two functions give reasonable bounds on $A(n, p, d)$ for "large" values of $d$.

Corollary 6. For all integers $n \geq d \geq 1$ we have

$$
L(n, d) \leq A(n, p, d) \leq U(n, d) .
$$

Furthermore, $A(d, p, d)=U(d, d)=0$ and for any integer $t \geq 2$ we have

$$
\lim _{d \rightarrow \infty} \frac{U(t d, d)}{L(t d, d)}=t+1
$$

Proof. It is implicit in [8] (see also [15]) that $L(n, d)$ is less than $A(n, p, d)$. So we shall concentrate on the upper bound, and on the case $d \neq 2$ (leaving to the reader the case $d=2)$. Certainly $A(n, p, 1)=U(n, 1)$, so let us suppose that $d \geq 3$. Clearly $A(d, p, d)=U(d, d)$. As an inductive hypothesis assume that $A(k, p, d) \leq U(k, d)$ for some $k \geq d$. For each group $G$ of order $p^{k+1}$ we can choose a central subgroup $Z$ of order $p$. The group $G$ is determined, up to an extension, by $Z$ and $G / Z$. In other words $G$ is determined by $Z, G / Z$, and an element of the second cohomology group $H^{2}\left(G / Z, \mathbb{Z}_{p}\right)$. The Universal Coefficient Theorem

$$
\operatorname{Ext}\left((G / Z)^{\mathrm{ab}}, \mathbb{Z}_{p}\right) \longleftrightarrow H^{2}\left(G / Z, \mathbb{Z}_{p}\right) \longrightarrow \operatorname{Hom}\left(M^{0}(G / Z), \mathbb{Z}_{p}\right)
$$

and Proposition 5(i) imply that

$$
\left|H^{2}\left(G / Z, \mathbb{Z}_{p}\right)\right| \leq\left|M^{0}(G / Z)\right| \cdot p^{d} \leq p^{(d(2 k-d+3)-2 k) / 2} .
$$

Therefore

$$
\begin{aligned}
A(k+1, p, d) & \leq U(k, d)+\log _{p}\left|H^{2}\left(G / Z, \mathbb{Z}_{p}\right)\right| \\
& \leq\left((d-1)\left(k^{2}-d^{2}\right)+(d(2 k-d+3)-2 k)\right) / 2 \\
& \leq\left((d-1)\left((k+1)^{2}-d^{2}\right)-d^{2}+2 d+1\right) / 2 \\
& \leq\left((d-1)\left((k+1)^{2}-d^{2}\right)\right) / 2 \\
& \leq U(k+1, d) .
\end{aligned}
$$

The upper bound of the proposition follows by induction. It is routine to verify the limit in the proposition.

Our proofs of the above theorems involve the $q$-exterior product $M \wedge^{q} N$ of two normal subgroups $M$ and $N$ of some group, a self-contained account of which can be found in [4]. We shall assume a familiarity with the definition of this $q$-exterior product, and with certain results from [4]. For $q=0$ the exterior product $M \wedge^{0} N$ is that of Brown and Loday [1].

It is shown in [4, p. 247] that $M \wedge^{q} N$ is finite if both $M$ and $N$ are finite. The following lemma relates the cardinality of the exterior square $Q \wedge^{q} Q$ to the above theorems. 
Lemma 7. Let $G$ be any group and set $Q=G / Z^{q}(G)$. Then:

(i) $\left|\gamma_{2}^{q} G\right| \leq\left|Q \wedge^{q} Q\right|$.

Moreover, there is a group $\widehat{G}$ such that $\widehat{G} / Z^{q}(\widehat{G}) \cong Q$ and $\gamma_{2}^{q} \widehat{G} \cong Q \wedge^{q} Q$.

(ii) $\left|M^{q}(G)\right|\left|\gamma_{2}^{q} G\right|=\left|G \wedge^{q} G\right|$.

Proof. Theorem 6 and Proposition 7 in [4] (the first of these results being due to [3]) yield the following diagram of solid homomorphisms:

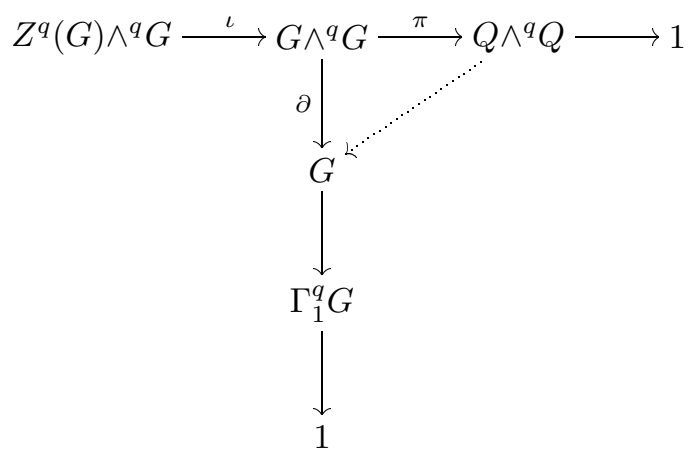

in which the row and column are exact, and in which $\operatorname{im}(\iota) \subseteq \operatorname{ker}(\partial)$. There is thus a surjection $Q \wedge^{q} Q \rightarrow \gamma_{2}^{q} G$ from which we deduce that $\left|\gamma_{2}^{q} G\right| \leq\left|Q \wedge^{q} Q\right|$.

Let $\mu: \widehat{G} \rightarrow Q$ be any projective $q$-crossed module with $\operatorname{im}(\mu)=Q$; the definition and existence of such an object is given in [4, p. 245]. Proposition 5 in [4] implies that $\gamma_{2}^{q} \widehat{G} \cong Q \wedge^{q} Q$. The proof of Proposition 16 (vii) in [4] implies that $\widehat{G} / Z^{q}(\widehat{G}) \cong$ $Q$.

Propositions 1 and 5 in [4] imply an exact sequence (which is also to be found in $[5])$

$$
1 \rightarrow M^{q}(G) \rightarrow G \wedge^{q} G \rightarrow G \rightarrow \Gamma_{1}^{q} G \rightarrow 1 .
$$

The equality $\left|M^{q}(G)\right|\left|\gamma_{2}^{q} G\right|=\left|G \wedge^{q} G\right|$ follows from the exactness of this sequence.

In the following lemma (and throughout the article) we use the symbol $\otimes$ to denote the usual tensor product of abelian groups considered as $\mathbb{Z}$-modules.

Lemma 8. (i) [1] Any normal inclusion $N \leq G$ gives rise to a natural exact sequence

$$
H_{3}^{0}(G) \rightarrow H_{3}^{0}(G / N) \rightarrow N \wedge^{0} G \rightarrow G \wedge^{0} G \rightarrow(G / N) \wedge^{0}(G / N) \rightarrow 1 .
$$

(ii) [4] Any pair of normal inclusions $N \leq G$ and $M \leq G$ with $M \subseteq N$ give rise to a natural exact sequence

$$
M \wedge^{0} G \rightarrow N \wedge^{0} G \rightarrow(N / M) \wedge^{0}(G / M) \rightarrow 1 .
$$

(iii) There is an isomorphism

$$
\left(\Gamma_{i}^{p} G\right) \wedge^{0}\left(G / \gamma_{i+1}^{p} G\right) \cong\left(\Gamma_{i}^{p} G\right) \otimes\left(\Gamma_{1}^{p} G\right)
$$

for $i \geq 2$.

(iv) For $q \geq p$ there is an exact sequence

$$
0 \rightarrow \gamma_{2}^{q}\left(M^{0}(G)\right) \rightarrow G \wedge^{0} G \rightarrow G \wedge^{q} G \rightarrow \Gamma_{1}^{0} G \rightarrow 0 .
$$


Proof. Part (i) can be found, for instance, in [1], and part (ii) is an easy modification of Proposition 7 in [4]. To prove (iii) note that $\Gamma_{i}^{p} G$ lies in the centre of $G / \gamma_{i+1}^{p} G$. As explained in [1], in this case the defining relations of the exterior product yield an isomorphism

$$
\Gamma_{i}^{p} G \wedge^{0}\left(G / \gamma_{i+1}^{p} G\right) \cong \Gamma_{i}^{p} G \otimes\left(G / \gamma_{i+1}^{p} G\right)^{\mathrm{ab}} /\left\langle x \otimes \bar{x} \mid x \in \Gamma_{i}^{p} G\right\rangle
$$

where $\bar{x}$ denotes the image of $x$ in the abelianization of $G / \gamma_{i+1}^{p} G$. The isomorphism of (iii) follows. The exact sequence of (iv) follows from Proposition 14 in [4].

Set $\varepsilon=0$ or $\varepsilon=p$. Let $Q$ be a $p$-group of order $p^{n}$. Let $c$ be the first integer such that then $\gamma_{c+1}^{\varepsilon} Q=1$.

Lemma 8(i) applied to the normal inclusion $\gamma_{2}^{\varepsilon} Q \leq Q$ yields the exact sequence

$$
\gamma_{2}^{\varepsilon} Q \wedge^{0} Q \stackrel{\alpha_{2}}{\longrightarrow} Q \wedge^{0} Q \longrightarrow \Gamma_{1}^{\varepsilon} Q \wedge^{0} \Gamma_{1}^{\varepsilon} Q \longrightarrow 1 .
$$

For $i \geq 2$ the normal inclusion $\gamma_{i+1}^{\varepsilon} Q \leq \gamma_{i}^{\varepsilon} Q$ in conjunction with Lemma 8(ii) yields the exact sequence

$$
\gamma_{i+1}^{\varepsilon} Q \wedge^{0} Q \stackrel{\alpha_{i+1}}{\longrightarrow} \gamma_{i}^{\varepsilon} Q \wedge^{0} Q \longrightarrow\left(\gamma_{i}^{\varepsilon} Q / \gamma_{i+1}^{\varepsilon} Q\right) \wedge^{0}\left(Q / \gamma_{i+1}^{\varepsilon} Q\right) \longrightarrow 1 .
$$

Sequences (1) and (2) in conjunction with Lemma 8(iii) imply

$$
\left|Q \wedge^{0} Q\right|=\frac{\left|\Gamma_{1}^{\varepsilon} Q \wedge^{0} \Gamma_{1}^{\varepsilon} Q\right|\left|\Gamma_{2}^{\varepsilon} Q \otimes \Gamma_{1}^{\varepsilon} Q\right| \cdots\left|\Gamma_{c-1}^{\varepsilon} Q \otimes \Gamma_{1}^{\varepsilon} Q\right|\left|\gamma_{c}^{\varepsilon} Q \otimes \Gamma_{1}^{\varepsilon} Q\right|}{\left|\operatorname{ker} \alpha_{2}\right|\left|\operatorname{ker} \alpha_{3}\right| \cdots\left|\operatorname{ker} \alpha_{c}\right|} .
$$

Recall that the tensor product of any two abelian groups can be computed from the following isomorphisms: $A \otimes(B \times C) \cong(A \otimes B) \times(A \otimes C), A \otimes B \cong B \otimes A$, and $C_{m} \otimes C_{n} \cong C_{h c f(m, n)}$ where $A, B, C$ are arbitrary abelian groups, and $C_{m}$ is the cyclic group of order $m$. Recall from [1] that for any abelian group $A$ there is an isomorphism $A \wedge^{0} A \cong A \otimes A /\langle a \otimes a: a \in A\rangle$. Thus, if

$$
\Gamma_{i}^{\varepsilon} Q \cong C_{p^{m_{i 1}}} \times C_{p^{m_{i 2}}} \times \cdots \times C_{p^{m_{i r}}}
$$

and if $M_{i j k}=\operatorname{minimum}\left(m_{i j}, m_{1 k}\right)$, then

$$
\begin{aligned}
& \log _{p}\left|\Gamma_{1}^{\varepsilon} Q \wedge{ }^{0} \Gamma_{1}^{\varepsilon} Q\right|=\sum_{1 \leq j<k \leq r} M_{1 j k}, \\
& \log _{p}\left|\Gamma_{i}^{\varepsilon} Q \otimes \Gamma_{1}^{\varepsilon} Q\right|=\sum_{1 \leq j, k \leq r} M_{i j k}, i \geq 2 .
\end{aligned}
$$

Note that each $\left|\Gamma_{i}^{\varepsilon} Q \otimes \Gamma_{1}^{\varepsilon} Q\right|$ is a maximum, for any given $\left|\Gamma_{i}^{\varepsilon} Q\right|=p^{m_{i}}$ and $\left|\Gamma_{1}^{\varepsilon} Q\right|=$ $p^{m_{1}}$, if and only if $\Gamma_{i}^{\varepsilon} Q$ and $\Gamma_{1}^{\varepsilon} Q$ are elementary abelian; in other words

$$
\begin{aligned}
& \left|\Gamma_{1}^{\varepsilon} Q \wedge^{0} \Gamma_{1}^{\varepsilon} Q\right| \leq p^{m_{1}\left(m_{1}-1\right) / 2}, \\
& \left|\Gamma_{i}^{\varepsilon} Q \otimes \Gamma_{1}^{\varepsilon} Q\right| \leq p^{m_{1} m_{i}}, i \geq 2 .
\end{aligned}
$$

Now (3) and (5) imply that

$$
\begin{aligned}
\left|Q \wedge^{0} Q\right| & \leq p^{m_{1}\left(m_{1}-1\right) / 2+m_{1} m_{2}+m_{1} m_{3}+\cdots m_{1} m_{c}} \\
& =p^{m_{1}\left(m_{1}-1+2 m_{2}+2 m_{3}+\cdots+2 m_{c}\right) / 2} \\
& =p^{m_{1}\left(2 n-m_{1}-1\right) / 2} .
\end{aligned}
$$


Note that if $\varepsilon=p$, then $m_{1}=d$ is the minimum number of generators needed for $Q$. Thus Theorem 1(i) follows from (6), the inequality of Lemma 7(i), and the exact sequence of Lemma 8(iv). For $i \geq 2$ Lemma 8(i) yields an exact sequence

$$
\begin{aligned}
H_{3}^{0}(Q) & \longrightarrow H_{3}^{0}\left(Q / \gamma_{i}^{\varepsilon} Q\right) \longrightarrow \gamma_{i}^{\varepsilon} Q \wedge^{0} Q \stackrel{\beta_{i}}{\longrightarrow} Q \wedge^{0} Q \\
& \longrightarrow\left(Q / \gamma_{i}^{\varepsilon} Q\right) \wedge^{0}\left(Q / \gamma_{i}^{\varepsilon} Q\right) \longrightarrow 1
\end{aligned}
$$

Now $\beta_{i}$ factors as

$$
\beta_{i}: \gamma_{i}^{\varepsilon} Q \wedge^{0} Q \stackrel{\alpha_{i}}{\longrightarrow} \gamma_{i-1}^{\varepsilon} Q \wedge^{0} Q \longrightarrow \cdots \stackrel{\alpha_{2}}{\longrightarrow} Q \wedge^{0} Q .
$$

So $\operatorname{ker} \beta_{i}=0$ for all $i \geq 2$ if and only if $\operatorname{ker} \alpha_{i}=0$ for all $i \geq 2$. This equivalence, together with Lemma 7(i) and our derivation of Theorem 1(i), imply Theorem 1(ii).

In order to prove Theorem 2 note that when $\varepsilon=0$ and $i \geq 2$ the homomorphism $\beta_{i}$ factors as

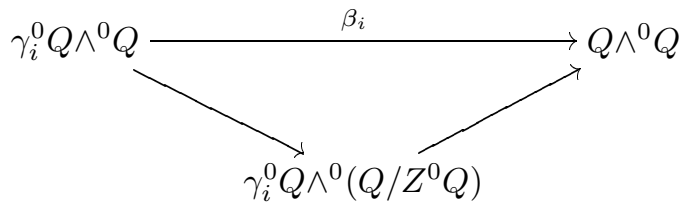

since the kernel of the canonical homomorphism $\gamma_{i}^{0} Q \wedge^{0} Q \rightarrow \gamma_{i}^{0} Q \wedge^{0}\left(Q / Z^{0} Q\right)$ is generated by the elements $x \wedge z$ with $x \in \gamma_{i}^{0} Q$ and $z \in Z^{0}(Q)$, and since $\beta_{i}(x \wedge z)=1$ for each such generator. (To prove the triviality of $\beta_{i}(x \wedge z)$, note that we can find an element $\tilde{x} \in Q \wedge{ }^{0} Q$ which maps canonically onto $x$. We have $\beta_{i}(x \wedge z)=$ $x \wedge z=\tilde{x}^{z} \tilde{x}^{-1}$ by [1]. But $z$ is central and thus acts trivially on $Q \wedge^{0} Q$. Therefore $\beta_{i}(x \wedge z)=\tilde{x}^{z} \tilde{x}^{-1}=1$.) This factorization, together with the arguments behind (3), yield the inequality

$$
\left|Q \wedge^{0} Q\right| \leq\left|\Gamma_{1}^{0} Q \wedge^{0} \Gamma_{1}^{0} Q\right|\left|\Gamma_{2}^{0} Q \otimes \Gamma_{1}^{0} \bar{Q}\right| \cdots\left|\Gamma_{c-1}^{0} Q \otimes \Gamma_{1}^{0} \bar{Q}\right|\left|\gamma_{c}^{\varepsilon} Q \otimes \Gamma_{1}^{0} \bar{Q}\right|
$$

where $\bar{Q}=Q / Z^{0}(Q)$.

The proof of Theorem 2 is similar to that of Theorem 1; the differences are that now $\varepsilon=0$, and that (7) can be used in place of (3).

Theorem 3 is an improvement on parts (i) and (ii) of Theorem 1 obtained by using Theorem 9 in [4] to identify certain non-trivial elements in $\operatorname{ker} \alpha_{i}$, and thus to estimate $\left|\operatorname{ker} \alpha_{i}\right|$ in (3).

Proposition 5 follows in the same fashion from (3), (7) and Lemma 7(ii).

To prove Theorem 4 suppose that $Q=G / Z^{0}(G)$ is of order $p^{n}$, and that $X=$ $\left\{x_{1}, \ldots, x_{d}\right\}$ is a subset of $G$ whose image in $Q$ generates $Q$, and which satisfies $\bar{b}(G)=b(X)$. Denote by $C_{i}$ the image in $Q$ of the centraliser $C\left(x_{i}\right)$ of $x_{i}$ in $G$. Denote by $U_{i}$ the cyclic subgroup of $Q$ generated by the image of $x_{i}$. The image of the canonical homomorphism $C_{i} \wedge{ }^{0} U_{i} \rightarrow Q \wedge{ }^{0} Q$ clearly lies in the kernel of the surjection $Q \wedge \wedge^{0} Q \rightarrow \gamma_{2}^{0} G$, and this observation is the basis of the proof. Set $\varepsilon=p$, let $V_{i}$ denote the cyclic summand of $Q / \gamma_{2}^{\varepsilon}(Q)$ generated by the image of $x_{i}$, and set $D_{i j}=C_{i} \cap \gamma_{j}^{\varepsilon}(Q)$. Note that the canonical homomorphisms

$$
\left(D_{i j} / D_{i j+1}\right) \otimes V_{i} \longmapsto \Gamma_{j}^{\varepsilon} Q \otimes \Gamma_{1}^{\varepsilon} Q
$$

are injective, and that

$$
\left|\left(D_{i 1} / D_{i 2}\right) \otimes V_{i}\right|\left|\left(D_{i 2} / D_{i 3}\right) \otimes V_{i}\right| \cdots\left|D_{i c} \otimes V_{i}\right|=\left|C_{i}\right|=p^{n-b\left(x_{i}\right)} .
$$


Combining these three observations with (3) yields

$$
\begin{aligned}
\left|\gamma_{2}^{0}(G)\right| & \leq \frac{\left|\Gamma_{1}^{\varepsilon} Q \otimes \Gamma_{1}^{\varepsilon} Q\right|\left|\Gamma_{2}^{\varepsilon} Q \otimes \Gamma_{1}^{\varepsilon} Q\right| \cdots\left|\Gamma_{c-1}^{\varepsilon} Q \otimes \Gamma_{1}^{\varepsilon} Q\right|\left|\gamma_{c}^{\varepsilon} Q \otimes \Gamma_{1}^{\varepsilon} Q\right|}{\prod_{1 \leq i \leq d}\left|\left(D_{i 1} / D_{i 2}\right) \otimes V_{i}\right|\left|\left(D_{i 2} / D_{i 3}\right) \otimes V_{i}\right| \cdots\left|\left(D_{i c} \otimes V_{i}\right)\right|} \\
& \leq p^{n d} / p^{n-b\left(x_{1}\right)+\cdots+n-b\left(x_{d}\right)} \\
& =p^{d \bar{b}(G)}
\end{aligned}
$$

as required.

To prove Theorem 1(iii) for $d=n \geq 2$ set $Q$ equal to the elementary abelian $p$-group on $n$ generators. Then $|Q|=\left|\Gamma_{1}^{q} Q\right|=p^{n}, R(Q, q)=0$, and $\left|Q \wedge^{q} Q\right|=$ $p^{(n(n-1) / 2)}$. Moreover, $Q \cong G / Z^{q}(G)$ for some $G$ by Proposition 16(vii) in [4]. Thus the second assertion of Lemma $7(\mathrm{i})$ establishes the existence of a group $\widehat{G}$ with $\widehat{G} / Z^{q}(\widehat{G}) \cong Q$ and $\left|\gamma_{2}^{q} \widehat{G}\right|=p^{(n(n-1) / 2)+R(Q, q)}$.

To prove Theorem 1(iii) for $d=n-1=2$ let us first note that for any abelian groups $A, B$ we can construct a semi-direct product

$$
A \square B=((A \otimes B) \times B) \rtimes Z
$$

in which the action of an element $a$ in $A$ on the action of an element $(\tau, b)$ in the direct product $(A \otimes B) \times B$ is given by

$$
{ }^{a}(\tau, b)=(\tau(a \otimes b), b) .
$$

If the elements of $A$ and $B$ all have order dividing $q$, then

$$
\gamma_{2}^{q}(A \square B) \cong A \otimes B .
$$

To prove Theorem 1(iii) for $d=n-1=2$ set $Q=C_{p} \square C_{p}$. Then $|Q|=p^{3},\left|\Gamma_{1}^{q} Q\right|=$ $p^{2}$, and one can readily verify that $\left|Q \wedge^{q} Q\right|=p^{3+R(Q, q)}=p^{(d(2 n-d-1) / 2)+R(Q, q)}$. Now $Q \cong G / Z^{q}(G)$ for some $G$ by Proposition 16 (vii) in [4]. Thus the second assertion of Lemma $7(\mathrm{i})$ establishes the existence of a group $\widehat{G}$ with $\widehat{G} / Z^{q}(\widehat{G}) \cong Q$ and $\left|\gamma_{2}^{q} \widehat{G}\right|=p^{(d(2 n-d-1) / 2)+R(Q, q)}$.

\section{REFERENCES}

[1] R. Brown and J.-L. Loday, Van Kampen theorems for diagrams of spaces, Topology 26 (1987), 311-335. MR 88m:55008

[2] J. Burns, G. Ellis, D. MacHale, P. Ó'Murchú, R. Sheehy, and J. Wiegold, Lower central series of groups with small upper central factors, Proc. Royal Irish Acad. (to appear).

[3] D. Conduché and C. Rodríguez-Fernádez, Nonabelian tensor and exterior products modulo $q$ and universal $q$-central relative extensions, J. Pure Applied Algebra 78, 139-160 (1992). MR 93b:20085

[4] G. Ellis, Tensor products and q-crossed modules, J. London Math. Soc. (2) 51 (1955), 243258. MR 96c:20096

[5] G. Ellis and C. Rodríguez-Fernádez, An exterior product for the homology of groups with integral coefficients modulo q, Cahiers Topologie Géom. Differentielle Catégoriques 30 (1989), 339-343. MR 90k:20087

[6] W. Gaschütz, J. Neubüser and Ti Yen, Über den Multiplikator von p-Gruppen, Math. Z. 100 (1967), 93-96. MR 36:272

[7] J. A. Green, On the number of automorphisms of a finite group, Proc. Royal Soc. A 237 (1956), 574-581. MR 18:464c

[8] G. Higman, Enumerating p-groups, I. Inequalities, Proc. London Math. Soc. (3) 10 (1960), 24-30. MR 22:4779

[9] M. R. Jones, Multiplicators of p-groups, Math. Z. 127 (1972), 165-166. MR 47:6851

[10] Some inequalities for the multiplicator of finite group, Proc. Amer. Math. Soc. 39 (1973), 450-456. MR 47:3524 
[11] Some inequalities for the multiplicator of a finite group II, Proc. Amer. Math. Soc. 45 (1974), 167-172. MR 50:4741

[12] W. Magnus, A. Karrass, and D. Solitar, Combinatorial group theory, Dover Publications, New York (1976). MR 54:10423

[13] I. Schur, Über die Darstellung der endlichen Gruppen durch gebrochene lineare Substitutionen, J. reine angew. Math. 127 (1904), 20-50.

[14] C. C. Sims, Enumerating p-groups, Proc. London Math. Soc. (3) 15 (1965), 151-166. MR 30:164

[15] M. Suzuki, Group Theory II, Grundlehren der mathematischen Wissenschaften 248, SpringerVerlag, 1986. MR 87e:20001

[16] M. R. Vaughan-Lee, Breadth and commutator subgroups of p-groups, J. Algebra 32 (1974), 278-285. MR 51:690

[17] J. Wiegold, Multiplicators and groups with finite central factor-groups, Math. Z. 89 (1965), 345-347. MR 31:3510

Department of Mathematics, University College, Galway, Ireland

E-mail address: graham.ellis@ucg.ie

Current address, September 1998 to June 1999: Max-Planck-Institut für Mathematik, GottfriedClaren-Straße 26, Bonn, Germany 